\title{
ST segment elevations and a pocket full of pus
}

\author{
Aniket S. Rali, Tarun Dalia, Aditya Rali, \\ Xiuxu Chen, Ivan Damjanov, Kamal Gupta
}

\begin{abstract}
Introduction: Very few cases of myocardial abscess are reported in medical literature. Although a rare disease entity in the developed countries, it carries a very high mortality rate and hence requires prompt diagnoses and treatment. Here we report a case of myocardial abscess diagnosed on autopsy. Case Report: A 47-year-old patient with complaints of progressively worsening shortness of breath, abdominal pain and chest pressure from three days. Electrocardiography showed ST segment elevations in leads I, II, III, aVR, aVL, V2-V6. Emergency room team suspected ST segment elevation myocardial infarction (STEMI) and cardiology department was consulted for emergent cardiac catheterization. Due to high clinical suspicion of severe sepsis, the decision was made to hold off on cardiac catheterization. The patient was transferred for emergent computed tomography scan to rule out intraabdominal infection. While in the scanner, patient developed pulseless electrical activity (PEA) arrest and passed away despite attempted
\end{abstract}

Aniket S. Rali', Tarun Dalia ${ }^{2}$, Aditya Rali ${ }^{3}$, Xiuxu Chen ${ }^{4}$, Ivan Damjanov $^{4}$, Kamal Gupta ${ }^{1}$

Affiliations: ${ }^{1}$ University of Kansas Medical Center, Division of Cardiovascular Diseases, Kansas City, USA; '2University of Kansas Medical Center, Department of Internal Medicine, Kansas City, USA; ${ }^{3}$ Emory University, School of Medicine; ${ }^{4}$ University of Kansas Medical Center, Department of Pathology, Kansas City, USA.

Corresponding Author: Aniket S. Rali MD, 3901 Rainbow Blvd MS 3006, Kansas City, KS 66160, USA; Email: arali@ kumc.edu

Received: 05 October 2017

Accepted: 08 November 2017

Published: 01 February 2018 resuscitation. Conclusion: Myocardial abscess should be considered in patients presenting with conduction defects and ST segment elevations on ECG in the setting of suspected bacteremia or endocarditis. Transthoracic echocardiogram, transesophageal echocardiogram and nuclear medicine scans can all contribute to timely diagnoses. Definitive treatment of myocardial abscess involves surgical drainage. Due to high mortality associated with this disease, prompt diagnoses and treatment is of utmost importance.

Keywords: STEMI, Myocardial Abscess, Pericarditis

\section{How to cite this article}

Rali AS, Dalia T, Rali A, Chen X, Damjanov I, Gupta K. ST segment elevations and a pocket full of pus. Int J Case Rep Images 2018;9:100880Zo1AR2018.

Article ID: 100880Zo1AR2018

$* * * * * * * * *$

doi: 10.5348/100880Zo1AR2018CR

\section{INTRODUCTION}

Myocardial abscess is predominantly seen in developing countries. Owing to early diagnoses and treatment of bacteremia, myocardial abscesses are very rare in developed countries with only a handful cases reported in medical literature. The incidence of myocardial abscess as determined in several autopsy studies ranges from $0.1-1.5 \%$ [1-3]. In the past, the most likely pathophysiology of bacterial myocardial abscess was believed to be preexisting bacteremia leading to 


\section{EDORiUM Journals}

spread of infection into the pericardium and myocardium [2]. However, recent reports suggest that most cases of myocardial abscess occur in conjunction with endocarditis of native or prosthetic valves [4]. Myocardial abscesses are often clinically silent and their presence is generally obscured by associated severe sepsis [3]. Herein, we describe a patient who presented with symptoms of chest and abdominal pain and was found to have myocardial abscess on autopsy.

\section{CASE REPORT}

A 47-year-old Caucasian male was presented to the emergency room with complaints of progressively worsening shortness of breath, abdominal pain and chest pressure for three days. His past medical history was significant for uncontrolled diabetes mellitus, hypertension, dyslipidemia, morbid obesity and polymicrobial Fourneir's gangrene with lower abdominal necrotizing fasciitis requiring several recent debridements. Wound cultures had grown Methicillin resistant Staphylococcus aureus (MRSA) and Enterococcus faecalis. Physical examination was notable for abdominal tenderness and multiple unhealed ulcers over left groin. He was tachypneic requiring 5 liters of supplemental oxygen but normotensive, nontachycardic and afebrile. Laboratory studies showed significant leukocytosis (WBC $28 \mathrm{~K} / \mathrm{UL}$ ), elevated lactate (5 mmol/L) and creatinine $(3.6 \mathrm{mg} / \mathrm{dL})$, decreased serum bicarbonate $(15 \mathrm{mmol} / \mathrm{L})$, hyperkalemia $(5.7$ $\mathrm{mmol} / \mathrm{L})$ and mildly elevated troponin (0.48 ng/mL). Electrocardiography showed ST segment elevations in leads I, II, III, aVR, aVL, V2-V6 (Figure 1A). Emergency room team suspected ST segment elevation myocardial infarction (STEMI) and cardiology department was consulted for emergent cardiac catheterization. Due to high clinical suspicion of severe sepsis, the decision was made to hold off on cardiac catheterization. Patient was transferred for emergent CT scan to rule out intraabdominal infection. While in the scanner, patient developed pulseless electrical activity (PEA) arrest and passed away despite attempted resuscitation.

Intra-CPR echo was suggestive of a moderate-sized pericardial effusion. Autopsy was requested which showed bacterial myocarditis with abscess formation involving the left ventricle (Figure $1 \mathrm{~B}-\mathrm{C}$ ) and fibrino-hemorrhagic pericarditis with $300 \mathrm{ml}$ of purulent pericardial effusion. Histology section from myocardium showed dense inflammation suggestive of myocardial abscess. (Figure 1D) Gram stain of the autopsy specimen showed gram positive bacterial colonies (Figure 1E).

\section{DISCUSSION}

Several aspects of this case are interesting including the presenting symptoms, ECG findings and the evolution

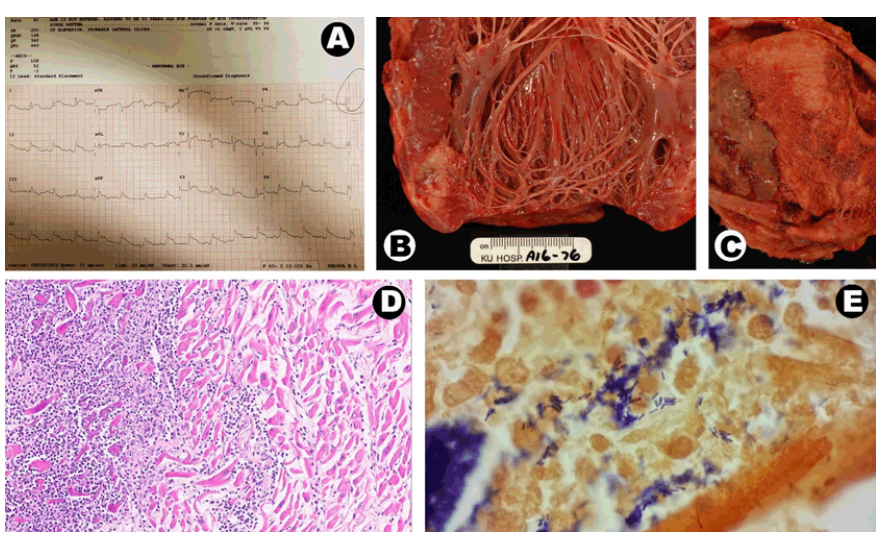

Figure 1: (A) Electrocardiography showing ST segment elevations in leads I, II, III, aVR, aVL, V2-V6. (B) Gross pathology sample showing abscess formation involving the left ventricle. (C) Gross pathology sample showing abscess formation involving the left ventricle. (D) Histopathology showing fibrino-hemorrhagic pericarditis (H\&E stain, x200). (E) Gram stain of the autopsy sample showing Gram Positive Rods (H\&E stain, x1000).

of differential diagnoses. Initially patient's symptoms of chest pain were attributed to acute coronary syndrome however laboratory and physical examination findings suggested an intra-abdominal pathology with possible extension of the inflammation into the pericardium and resultant pericarditis. Diffuse ST segment elevations on ECG and presence of pericardial effusion on bedside echo were also suggestive of this.

We believe the etiology of PEA arrest to be multifactorial including acidosis, hypoxia and hyperkalemia. Cardiac tamponade could not be ruled out. This case highlights the importance of integration of all available clinical data and maintaining a broad differential diagnoses in reaching a rare diagnosis such as myocardial abscess.

Myocardial abscess is a rare and lethal disease that requires prompt diagnoses and treatment. This should be included in the differential in any patient with known or suspected bacteremia and signs of cardiac involvement. In addition to treatment with intra-venous antibiotics, definitive treatment of myocardial abscess involves surgical drainage.

In addition to bacteremia, and bacterial endocarditis, myocardial abscess can also occur as a complication of other clinical conditions such as acute myocardial infarction, trauma with penetrating injuries, following invasive cardiac procedures, left ventricular aneurysm infection, atrial myxoma infection and HIV-associated [5]. In our patient, there was no evidence of endocarditis on autopsy.

\section{CONCLUSION}

Myocardial abscess should be considered in patients presenting with conduction defects and ST segment 
elevations on electrocardiography in the setting of suspected bacteremia or endocarditis. Transthoracic echocardiogram, transesophageal echocardiogram and nuclear medicine scans can all contribute to timely diagnoses. Definitive treatment of myocardial abscess involves surgical drainage. Due to high mortality associated with this disease, prompt diagnoses and treatment is of utmost importance.

\section{REFERENCES}

1. Flaxman N. Myocardial abscess. JAMA 1943;122(12):804-6.

2. Sanson J, Slodki S, Gruhn JG. Myocardial abscesses. Am Heart J 1963 Sep;66:301-8.

3. Anguera I, Quaglio G, Ferrer B, Sudden death in Staphylococcus aureus-associated infective endocarditis due to perforation of a freewall myocardial abscess. Scand $J$ Infect Dis 2001;33(8):622-5.

4. González Vílchez FJ, Martín Durán R, Delgado Ramis $\mathrm{C}$, et al. Active infective endocarditis complicated by paravalvular abscess: Review of 40 cases. [Article in Spanish]. Rev Esp Cardiol 1991 May;44(5):306-12.

5. Chakrabarti J. Diagnostic evaluation of myocardial abscesses: A new look at an old problem. Int J Cardiol 1995 Dec;52(3):189-96.

$* * * * * * * * *$

\section{Author Contributions}

Aniket S. Rali - Substantial contributions to conception and design, Acquisition of data, Analysis and interpretation of data, Drafting the article, Revising it critically for important intellectual content, Final approval of the version to be published

Tarun Dalia - Substantial contributions to conception and design, Drafting the article, Revising it critically for important intellectual content, Final approval of the version to be published
Aditya Rali - Substantial contributions to conception and design, Drafting the article, Revising it critically for important intellectual content, Final approval of the version to be published

Xiuxu Chen - Substantial contributions to conception and design, Drafting the article, Revising it critically for important intellectual content, Final approval of the version to be published

Ivan Damjanov - Substantial contributions to conception and design, Drafting the article, Revising it critically for important intellectual content, Final approval of the version to be published

Kamal Gupta - Substantial contributions to conception and design, Drafting the article, Revising it critically for important intellectual content, Final approval of the version to be published

\section{Guarantor of Submission}

The corresponding author is the guarantor of submission.

\section{Source of Support}

None

\section{Consent Statement}

Written informed consent was obtained from the patient for publication of this case report.

\section{Conflict of Interest}

Authors declare no conflict of interest.

\section{Copyright}

(C) 2018 Aniket S. Rali et al. This article is distributed under the terms of Creative Commons Attribution License which permits unrestricted use, distribution and reproduction in any medium provided the original author(s) and original publisher are properly credited. Please see the copyright policy on the journal website for more information.
Access full text article on other devices

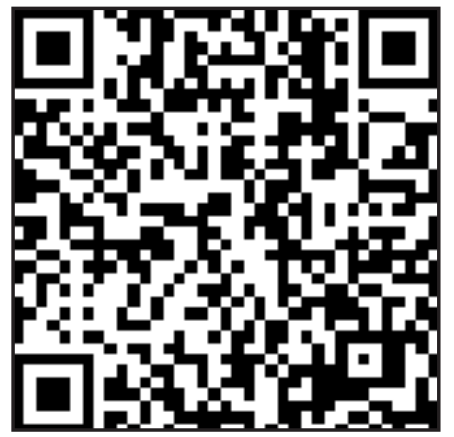

Access PDF of article on other devices

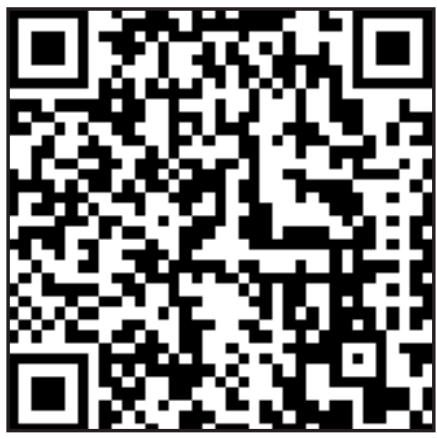

